\title{
Laparoscopy in Management of Blunt and Penetrating Abdominal Trauma: Single Center Experience
}

\author{
Adel Morad Abdallah, MD; Ahmed Zaki Gharib, MD \\ Department of General Surgery, Faculty of Medicine, October 6 University, Giza, Egypt
}

Introduction: Exploratory laparotomy in either blunt or penetrating abdominal trauma with suspected intra-abdominal injuries is associated with a high negative (non-therapeutic) laparotomy rate and a high procedure-related morbidity. In abdominal trauma, laparoscopy may be used either as a diagnostic or therapeutic tool in hemodynamically stable patients. Laparoscopy can avoid unnecessary (non-therapeutic) laparotomy and may allow laparoscopic repair of these injuries. Diagnostic laparoscopy can reliably rule out a significant intra-abdominal injury in patients with equivocal abdominal examination following trauma. In sharp abdominal trauma laparoscopy has been shown to be very effective in determining violation of the peritoneal cavity by tangential wounds.

Patients and methods: 38 patients with abdominal trauma were enrolled for this study. Exclusion criteria for all patients included; hemodynamic instability, clinical or radiological evidence of major abdominal organs or vascular injuries, posterior abdominal wall penetrating injuries, bowel evisceration, evidence of thoracic injuries (as pneumothorax), or head injuries. Pneumo-peritoneum is achieved with low $\mathrm{CO}_{2}$ flow and maintained at low pressures $(10-12 \mathrm{mmHg})$. We started by exploration of abdominal cavity and suction of the free blood from the peritoneal cavity. In sharp injuries, inspection of the peritoneal membrane was done for evidence of penetration. Small hepatic and splenic hematomas were left undisturbed. Small and accessible tears ware sutured. Deep or inaccessible liver and splenic tears needed conversion to laparotomy. Small intestinal simple tears were sutured laparoscopically while complex tears needed laparotomy conversion. Left colonic tears needed laparotomy conversion and colonic diversion.

Results: Patients with blunt trauma (26 patients), in whom laparoscopic management was completed were 18 patients (69\%). 5 patients (26\%) of the later, had negative laparoscopic exploration, for which the operations were terminated, 6 patients $(37 \%)$ had retroperitoneal hematomas with free hemo-peritoneum, 2 cases $(10.5 \%)$ showed only right hepatic lobe small sub-capsular hematomas, 3 cases (16\%) showed small liver tears, and 2 cases (10.5\%) showed small splenic sub-capsular hematomas and small superficial tear. All the previous injuries were left undisturbed with laparoscopic suturing of the 2 cases with liver tears. 12 cases $(31.5 \%)$ with sharp penetrating anterior abdominal wall trauma were included in this study. The cases with penetrating abdominal trauma that could be completed laparoscopically were 8 cases (67\%) and those who needed laparotomy conversion were 4 cases (33\%). The mean operative time in the cases that were completed laparoscopically was 85 minutes and in the laparotomy converted group was 67 minutes. Analysis of postoperative pain in all patients was done using the Numeric Rating Scale (NRS) for pain. Pain was subjectively more severe and statistically different in the laparotomy group than laparoscopic group. The range of hospital stay in the laparoscopy group was 2 to 5 days, and in the laparotomy group it was from 6 to 8 days. No cases in the laparoscopy group showed wound infection; while it was 3 cases (25\%) in the laparotomy converted group. Long term follow up showed one case (8.3\%) in the laparotomy group developed incisional hernia related to the main abdominal wound, while no similar case was detected in the laparoscopy completed group.

Conclusion: The evaluation and management of abdominal trauma are multi-factorial. Careful selection of trauma patients, high index of suspicion, and a low threshold for laparotomy will provide them the benefits of laparoscopic management and reduce the rates and morbidity of unnecessary laparotomy.

\section{Introduction}

Worldwide, the death rate from trauma in general is increasing and the deaths are mostly of young age groups. The principles of managing blunt and penetrating abdominal injuries have been well established. Control of life-threatening and complex intra-abdominal injuries requires rapid abdominal exploration that can only be accomplished through laparotomy incision as the time required to perform a laparoscopic procedure with its limited exposure, makes laparoscopy unrealistic option in those group of trauma patients.

Exploratory laparotomy in either blunt or penetrating abdominal trauma with suspected intra-abdominal injuries is associated with a high 
negative (non-therapeutic) laparotomy rate and a high procedure-related morbidity ( $41 \%$ according to Renz). ${ }^{1}$ With the advent of fine laparoscopic instruments, laparoscopic procedures have become standards for the treatment of many surgical conditions, being able to minimize impact of surgery, to reduce postoperative pain, hospital stay and time to recover, and to improve cosmetic outcome. The use of laparoscopy as an aid in the diagnosis of abdominal trauma was described in 1977.2,3 The use of laparoscopy in abdominal trauma has increased exponentially after a study done by Cuschieri who compared diagnostic peritoneal lavage with laparoscopy in blunt abdominal trauma patients. ${ }^{4}$ In abdominal trauma, laparoscopy may be used either as a diagnostic or therapeutic tool. Laparoscopy can avoid unnecessary (non-therapeutic) laparotomy and may allow laparoscopic repair of these injuries. ${ }^{5}$ Laparoscopy and other minimally invasive surgical techniques can be applied when a primarily intraabdominal injury is suspected in a hemodynamically stable patient. ${ }^{6}$ Hemodynamic stability sufficient to consider laparoscopy in a trauma patient was defined by Choi and Lim as a systolic blood pressure $>100 \mathrm{mmHg}$ and diastolic $>60 \mathrm{mmHg}$, a heart rate $<110$ beats per minute, and crystalloid resuscitation requirements of $<2$ L. ${ }^{6}$ Despite new diagnostic techniques such as ultrasonography and computed tomography, it seems that an acute abdominal condition (including blunt abdominal trauma) presents a situation in which a surgeon dares to open an abdomen, that might be delayed in many cases without a clear diagnosis and subjecting such patients to increased morbidity and mortality rates. ${ }^{7}$ The decision in favor of surgery or non-operative conservative treatment in blunt and penetrating abdominal trauma requires precise diagnosis that is not always possible with imaging techniques, whereby there is great danger that an injury to the diaphragm or intestines may be overlooked. ${ }^{8}$ Meta-analysis of sixty-four studies including 9058 patients with abdominal trauma in whom laparoscopy was used as a screening, diagnostic, or therapeutic tool showed significant reductions in the incidence of postoperative complications, perioperative mortality rates and hospital stays. ${ }^{9}$

If morbidity and mortality are to be reduced in patients with penetrating abdominal trauma, first priority goes to prompt and accurate determination of peritoneal penetration and identification of the need for surgery. In this setting, laparoscopy may have an important impact on the rate of negative or non-therapeutic laparotomies. ${ }^{10}$ Laparoscopy has been shown to be very effective in determining violation of the peritoneal cavity by tangential wounds and in diagnosis of anterior peritoneal penetration from stab wounds. ${ }^{11}$
Aim of our study was to evaluate the visibility and outcome of laparoscopy in managing selected cases of blunt and penetrating abdominal trauma in hemodynamically stable patients.

\section{Patients and methods}

38 patients with abdominal trauma ( 26 with blunt and 12 with penetrating trauma) were enrolled for this study in October 6 University Hospital in the period between July 2012 and July 2015. Inclusion criteria in blunt trauma patients included hemodynamic stability (pulse and blood pressure) with abdominal signs of hemo-peritoneum or peritoneal irritation with lack of major abdominal organs lacerations in abdominal ultrasound and Computerized tomography (C.T.) scans or lack of results clarity of these radiological investigations.

Selection criteria in penetrating abdominal trauma included abdominal stab wounds with proved or equivocal penetration of the peritoneum in hemodynamically stable patients with no or unclear evidence of vascular or major visceral injuries detected clinically and or radiologically by C.T. or ultrasound.

Exclusion criteria for all patients included; hemodynamic instability, radiological evidence of major abdominal organs or vascular injuries, posterior abdominal wall penetrating injuries, bowel evisceration, evidence of thoracic injuries (as pneumothorax), or head injuries, and in addition to the general contraindications to any laparoscopic procedure as previous laparotomy.

All abdominal trauma patients presented to the emergency unit were thoroughly evaluated by $A B C D$ management system to select the patients fulfilling the inclusion criteria of the study. All patients thereafter were subjected to detailed history about type, timing, and mode of impact of trauma. Full general examination was done to detect associated injuries or fractures. Clinical abdominal examination was done followed by Focused Abdominal Sonography in Trauma (FAST) and C.T. scans to detect cases indicated for laparoscopic exploration and to exclude others with exclusion criteria. Plain X- ray abdomen erect was also done to detect hollow viscus injury. Chest $\mathrm{X}$ - ray was ordered to detect associated thoracic injuries in suspected cases. Complete blood count was done for all patients at admission and was regularly repeated. Base line routine laboratory investigations were also done for all patients as serum GPT, GOT, BUN, creatinine, and amylase, All patients were admitted to intensive care unit preoperatively.

\section{Methods}

Preoperative preparations of patients scheduled for 
laparoscopic exploration were as follows: Insertion of central venous line, urinary catheter and nasogastric tube, intravenous fluid administration and blood preparation reserved for surgery.

All patients were operated on in October 6 university hospital under general endo-tracheal anesthesia by the same surgery team. The patients were laid in supine position. Abdominal insufflation was done using $\mathrm{CO}_{2}$ gas introduced either through Verres' needle or open technique at the umbilicus. Pneumo-peritoneum was achieved with low $\mathrm{CO}_{2}$ flow rates and maintained at low pressures $(10-12 \mathrm{mmHg})$. Low flow rate allowed timely detection of a tension pneumothorax (increased ventilatory pressures and/or hypotension). In cases of penetrating wounds, air leaks through the skin needed to be controlled with sutures before insufflations. The optical port $10 \mathrm{~mm}$ was introduced at the umbilicus. The laparoscope was introduced with either lens 0 or 30 according to the situation intra-abdominally. Abdominal exploration was done to determine visceral injuries. Two additional ports $5 \mathrm{~mm}$ or $10 \mathrm{~mm}$ were introduced at both sides of the umbilical port with modification of the levels according to the need. We started by exploration of abdominal cavity and suction of the associated hemo-peritoneum (Figure 1). In sharp injuries, inspection of the peritoneal membrane was done for evidence of penetration (Figure 2). We explored the inferior surface of the diaphragm for tears. Next, we explored the liver and spleen with the patient in anti-Trendlenberg's position and table tilt to one side to allow falling away of the intestinal loops. Small sub-capsular liver hematomas were left alone undisturbed. Small superficial non-actively bleeding liver tears were either left alone or packed with sugicell sheets (Figure 3). Accessible bleeding liver tears were sutured using absorbable sutures intracorporeally. Uncontrollable or inaccessible liver tears needed conversion to midline laparotomy and were controlled by suturing or packing with surgicell. Patients with small sub-capsular splenic hematomas or small controlled superficial tear were left undisturbed (Figure 4). Large, deep, multiple or actively bleeding splenic tears (Figure 5) needed conversion to laparotomy through midline incision and performing formal splenectomy. Laparoscopic exploration was then directed towards the stomach and small intestinal loops starting from the ligament of Treitz to the terminal ileum using the hand-over-hand technique with small fine bowel graspers introduced from the 2 side ports, with inspection of the mesentery for evidence of hematomas or tears (Figure 6). In some cases, methylene blue dye was administered via the nasogastric tube to help to identify proximal bowel injuries. Small, non-bleeding mesenteric tears or hematomas were left undisturbed.
Small intestinal tears were sutured laparoscopically using non-absorbable sutures (Figure 7). Patient with irregular and multiple small intestinal tears needed conversion to midline laparotomy and performing resection and anastomosis of the injured intestinal segment using $3 / 0$ absorbable suture material.

Exploration then proceeded to the colon with the patient tilted to lateral positions during inspection of the right colon, left colon and para-colic gutters.

Finally we inspected the pelvis for hematomas, free blood or evidence of pelvic organs injuries while the patient was in Trendlenberg's position.

The patient with injury of the sigmoid colon underwent laparotomy conversion and resection of the injured colonic segment and performing proximal colostomy and closure of the distal stump (Hartman's procedure). Patients in whom retroperitoneal hematomas were detected laparoscopically with no other lesions were left undisturbed. Suction and irrigations of the peritoneal cavity using normal saline for the associated hemo-peritoneum and leaving wide tube drains in the abdomen and pelvis were done for all cases whether completed laparoscopically or converted to laparotomy.

Operative findings, types of injury, management, rate of laparotomy conversions and operative time were all statistically evaluated. Postoperative events that were statistically recorded were hemodynamic stability, analgesics and narcotic requirements, hospital stay, early and late complications, and the return to full activity.

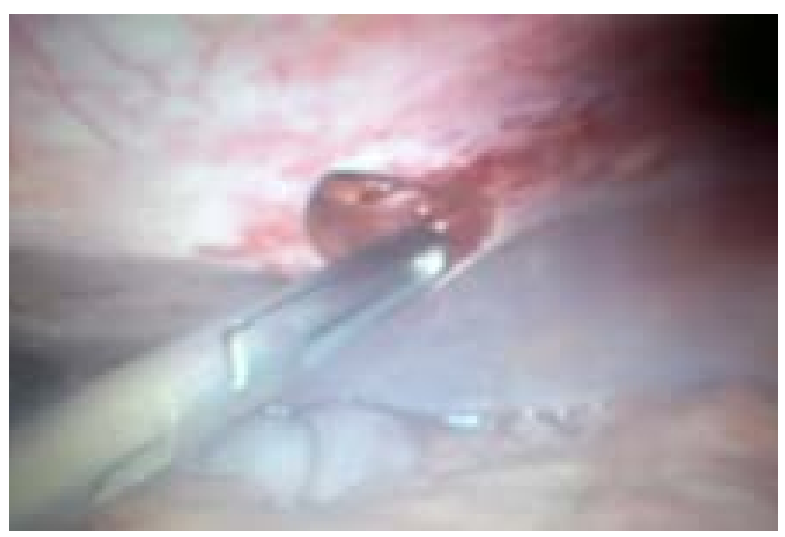

Fig 1: Penetrating wound in the anterior abdominal parities. 


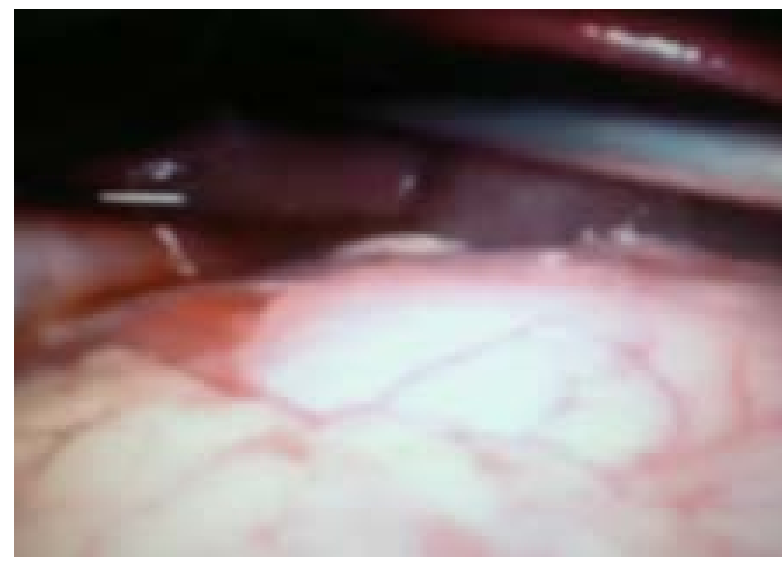

Fig 2: Hemoperitoneum.

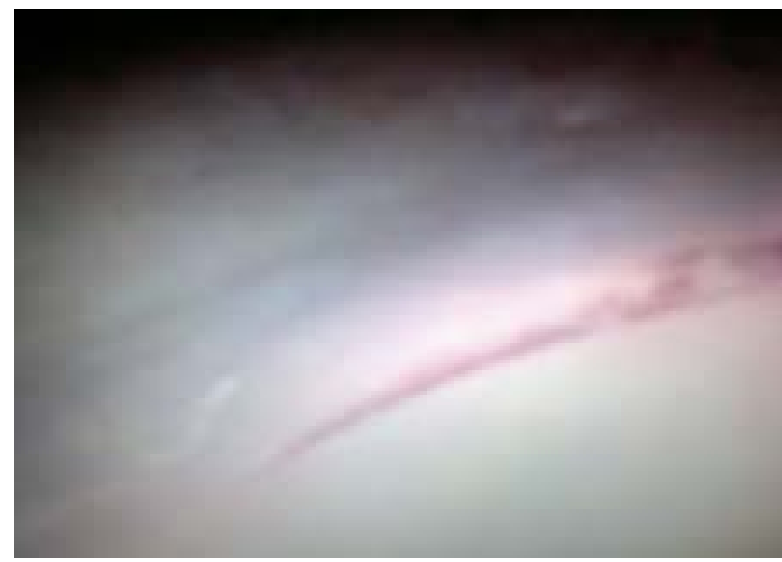

Fig 3: Right lobe hepatic tear.

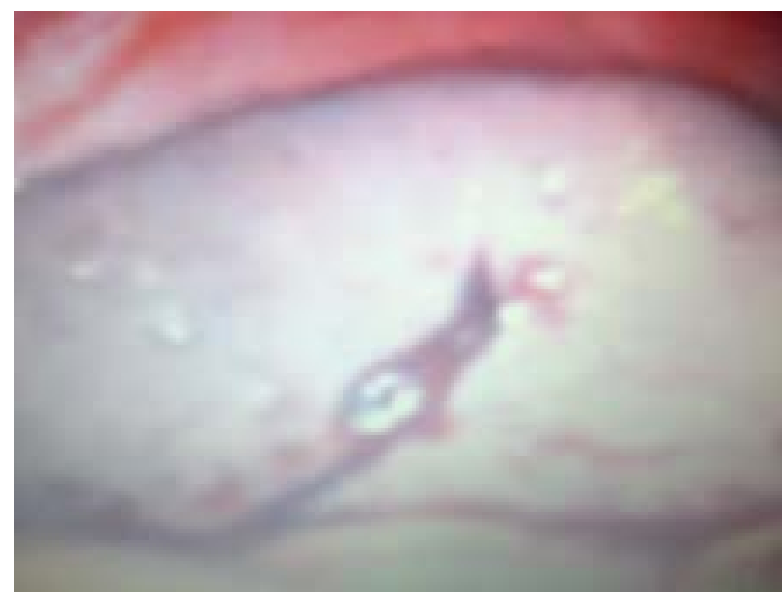

Fig 4: Dry splenic tear.

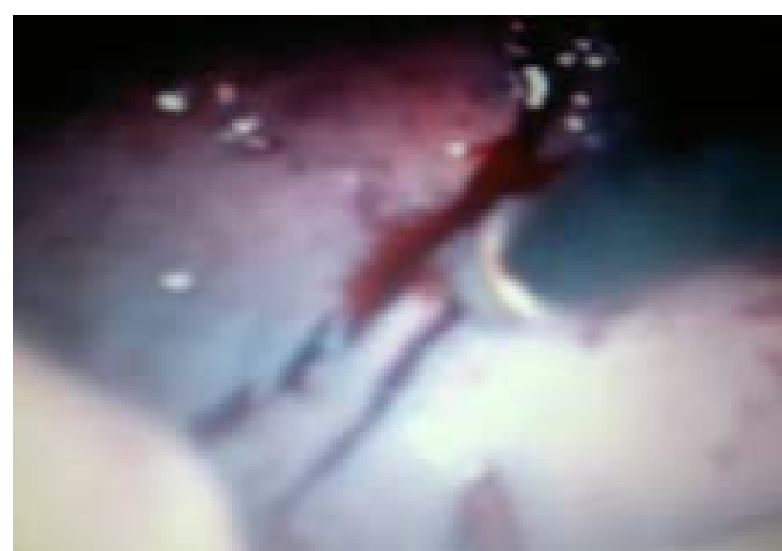

Fig 5: Actively bleeding splenic tears.

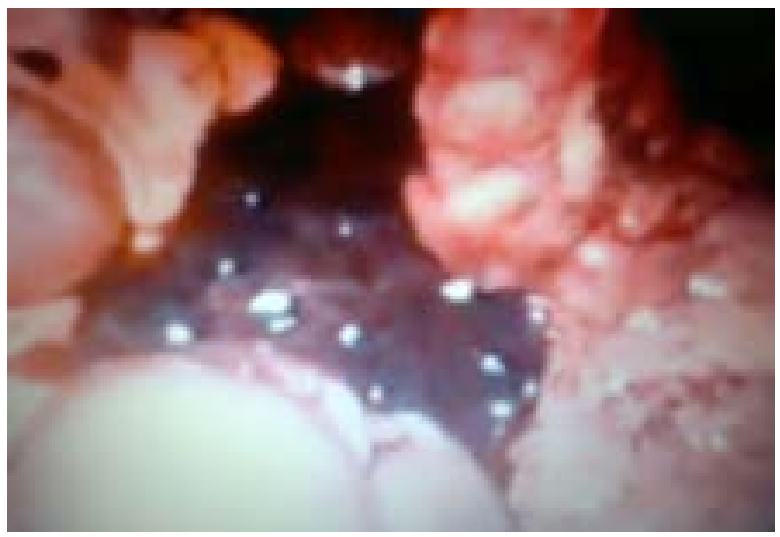

Fig 6: Mesenteric hematoma.

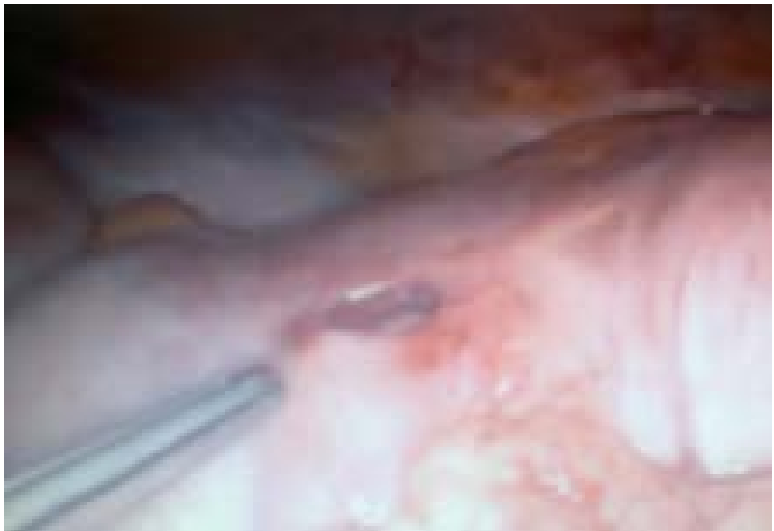

Fig 7: Simple intestinal tear.

\section{Results}

38 patients with abdominal trauma ( 26 with blunt and 12 with penetrating trauma) filling the inclusion criteria were the target of this study (Figure 8). The demographic data distribution of the patients is shown in Figure 9 and Table 1. The types of trauma and their relations to age groups are shown in Figure 10. The later shows that the age group mostly exposed to trauma was between 18 and 40 years (28 patients, 73.6\%). Motor car accidents were the main cause of blunt trauma, presented in 17 patients $(60.7 \%)$ (Figure 11). All patients with sharp penetrating trauma were due to abdominal stabs (Figure 12). Patients with blunt trauma (26 patients), in whom laparoscopic management was completed were 18 patients $(69 \%)$ (Figure 11). Five patients (26\%) of the later, had negative laparoscopic exploration, for which the operations were terminated. Six patients $(37 \%)$ had retroperitoneal hematomas with free hemo-peritoneum and 2 of them had associated small accessible liver tears. Two cases (10.5\%) showed only right hepatic lobe small sub- capsular hematomas with some free peritoneal blood. Three cases $(16 \%)$ showed small liver tears ( 2 in the right and one in the left lobe); 2 of these tears were accessible and could be sutured laparoscopically and one was small with no evidence of bleeding, and was left unsutured. Two cases (10.5\%) showed small splenic sub-capsular hematomas and small superficial tear that was dry, and both 
were left undisturbed.

Patients with blunt trauma in whom conversion to laparotomy was done were 8 cases $(30.7 \%)$ as shown in Figure 11. Three cases $(11.5 \%)$ showed inaccessible and deep tears in right lobe of the liver that could not be sutured laparoscopically, and had undergone laparotomy conversion, and then were sutured. Three cases (11.5\%) with deep or multiple splenic tears and retro-peritoneal hematomas needed laparotomy conversion and splenectomy. Two cases (7.7\%) showed unsatisfactory laparoscopic exploration with small amount of free hemo-peritoneum and underwent laparotomy with no specific injuries detected in one case, while retroperitoneal hematoma was detected in the other. Hence the total cases with blunt trauma that were completed laparoscopically were 18 cases $(69 \%)$ and those who needed conversion to laparotomy were 8 cases $(31 \%)$.

12 cases (31.5\%) with sharp penetrating anterior abdominal wall trauma were included in this study. Figure 12 shows the types of injuries and the way of completion of the procedure. Three cases (25\%) showed negative laparoscopic exploration (only the sites of penetration in the peritoneal membrane were evident with no other associated lesions) and the procedures were terminated.

Three patients $(25 \%)$ showed small mesenteric hematomas that were left undisturbed and little amount of hemo-peritoneum that was aspirated and the procedures were complete laparoscopically. 2 patients $(16.6 \%)$ had single tear in the ileum at the anti-mesenteric border that could be repaired laparoscopically using absorbable suture material. One case $(8.3 \%)$ showed multiple small intestinal tears that were difficult to be completed laparoscopically and necessitated conversion to laparotomy and resection anastomosis. One case $(8.3 \%)$ showed small sigmoid colon injury that necessitated conversion to laparotomy and performing Hartmann's procedure. Two cases $(16.6 \%)$ with hemo-peritoneum needed laparotomy conversion due to unsatisfactory laparoscopic explorations. One of the later showed bleeding from small vessels in the mesentery that was controlled by ligation and cauterization, while the other showed negative laparotomy exploration. Hence, total cases with penetrating abdominal trauma that could be completed laparoscopically were 8 cases $(67 \%)$ and those who needed laparotomy conversion were 4 cases (33\%).

The total cases (blunt and sharp trauma) that could be completed successfully laparoscopically were 27 cases $(71 \%)$ and the converted cases were 11 cases (29\%) (Figure 13).

The mean operative time in the operations that were completed laparoscopically was 85 minutes (range, 60 to 115 minutes) and in the laparotomy converted group was 67 minutes (range, 45 to 80 minutes) (Table 1).

All patients in both groups through the postoperative course were hemodynamically stable, the abdominal conditions were improved and hemoglobin $\%$ and hematocrit values were improved. There were no specific operative or early postoperative complications in all patients. Analysis of postoperative pain in all patients was done using the Numeric Rating Scale (NRS) for pain. Pain was subjectively more severe and statistically different in the laparotomy group than the laparoscopic group (Table 2). The administration of narcotics was more in the converted group than the laparoscopy group. Ambulation of patients was early in the laparoscopy group, average within one day, while it was average 2 days in the laparotomy group.

The range of hospital stay in the laparoscopy group was 2 to 5 days, and in the laparotomy group it was from 6 to 8 days (Table 3). The delay in discharge in the laparoscopy group was in the cases with small intestinal injuries while the delay in the laparotomy group was in the cases with intestinal injuries and wound infection. No cases in the laparoscopy group showed wound infection; while it was 3 cases $(25 \%)$ in the laparotomy converted group (Table 4). Long term follow up showed one case $(8.3 \%)$ in the laparotomy group developed incisional hernia related to the main abdominal wound, while no similar cases were detected in the laparoscopy completed group (Table 5).

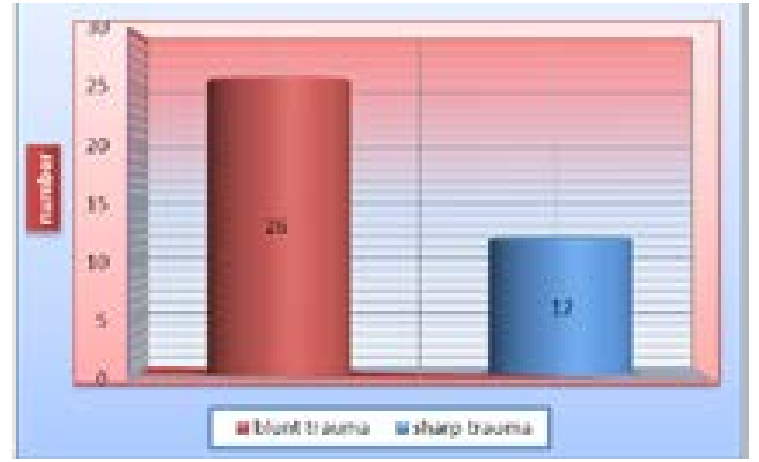

Fig 8: Types of abdominal trauma.

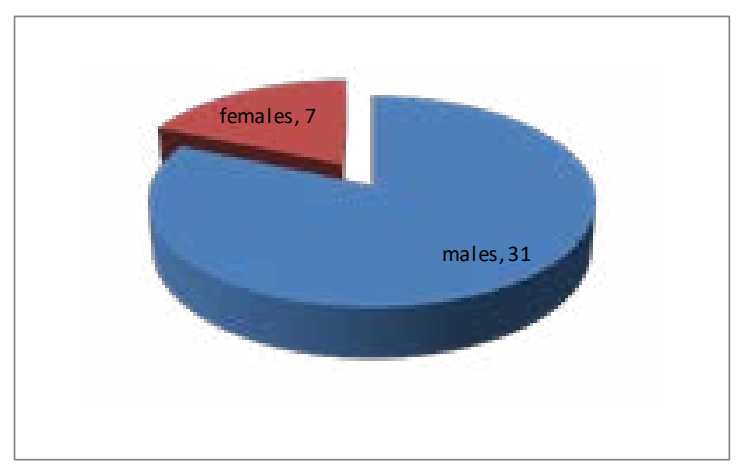

Fig 9: Sex distribution. 
Table 1: Demographic data

\begin{tabular}{lcccc}
\hline \multirow{2}{*}{ Type of trauma } & Mean age $(\mathbf{y})$ & \multicolumn{2}{c}{ Sex } & Total (n) \\
\cline { 3 - 4 } & & $\begin{array}{c}\text { Males } \\
\text { \% }\end{array}$ & $\begin{array}{c}\text { Females } \\
\text { \% }\end{array}$ & \\
\hline Blunt & 37 & $21(55)$ & $5(13)$ & 26 \\
Penetrating & 25 & $10(26)$ & $2(5)$ & 12 \\
\hline
\end{tabular}

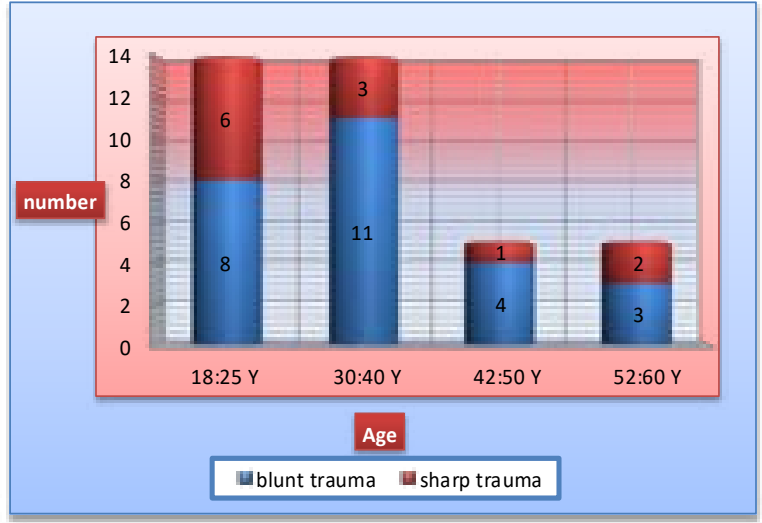

Fig 10: Age and types of trauma.

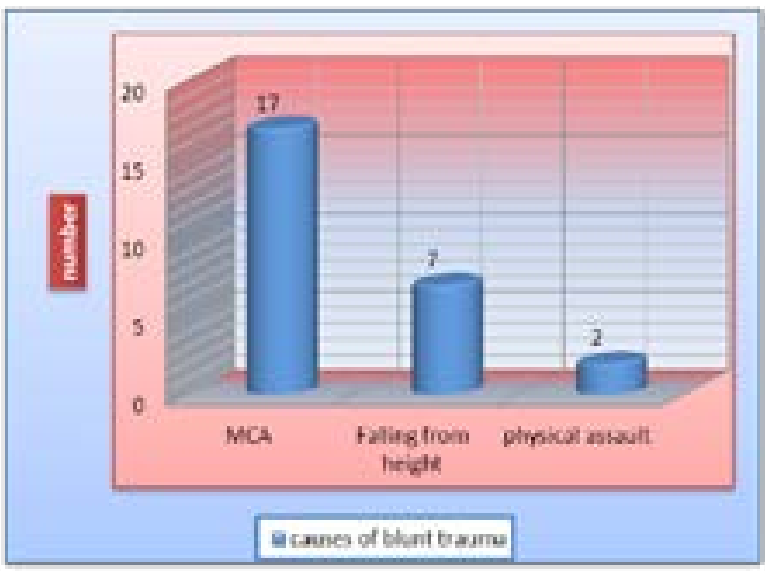

Fig 11: Causes of blunt trauma.

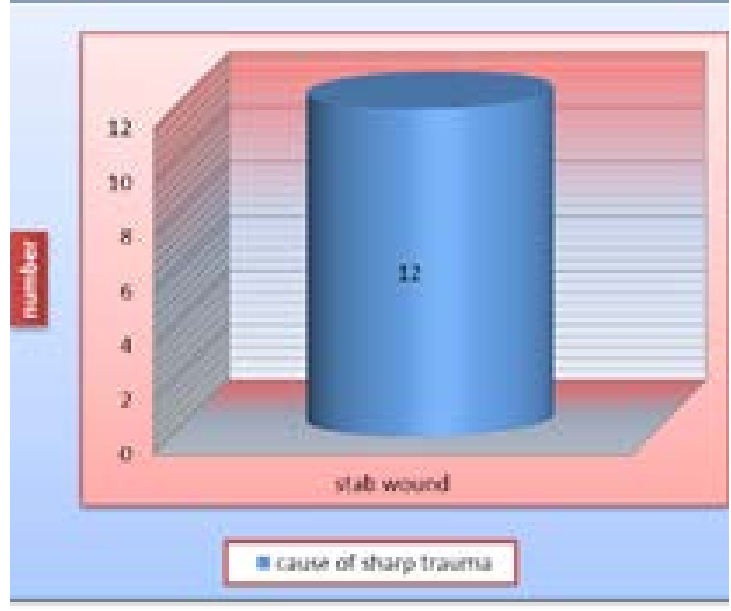

Fig 12: Cause of sharp trauma.

Table 2: Types of injuries and way of management

\begin{tabular}{lccccccc}
\hline \multirow{2}{*}{ Types of injury } & $\begin{array}{c}\text { Blunt trauma } \\
(\mathbf{n})\end{array}$ & $\begin{array}{c}\text { Penetrating } \\
\text { trauma (n) }\end{array}$ & Total (n) & & \multicolumn{3}{c}{ Management } \\
\hline Retroperitoneal hematoma & 6 & 0 & 6 & 6 & 16 & 0 & 0 \\
Splenic injuries & 5 & 0 & 5 & 2 & 5 & 3 & 8 \\
Liver injuries & 8 & 0 & 8 & 5 & 13 & 3 & 8 \\
Isolated hemoperitoneum & 0 & 2 & 2 & 0 & 0 & 2 & 5 \\
Intestinal tears & 0 & 4 & 4 & 2 & 5 & 2 & 5 \\
Mesenteric tears & 0 & 3 & 3 & 3 & 8 & 0 & 0 \\
Unsatisfactory & 2 & 0 & 2 & 0 & 0 & 2 & 5 \\
Negative laparoscopy & 5 & 3 & 8 & 8 & 21 & 0 & 0 \\
Total & 26 & 12 & 38 & 26 & 69 & 12 & 31 \\
\hline
\end{tabular}

T.L., Therapeutic laparoscopy

L.C., Laparotomy conversion 


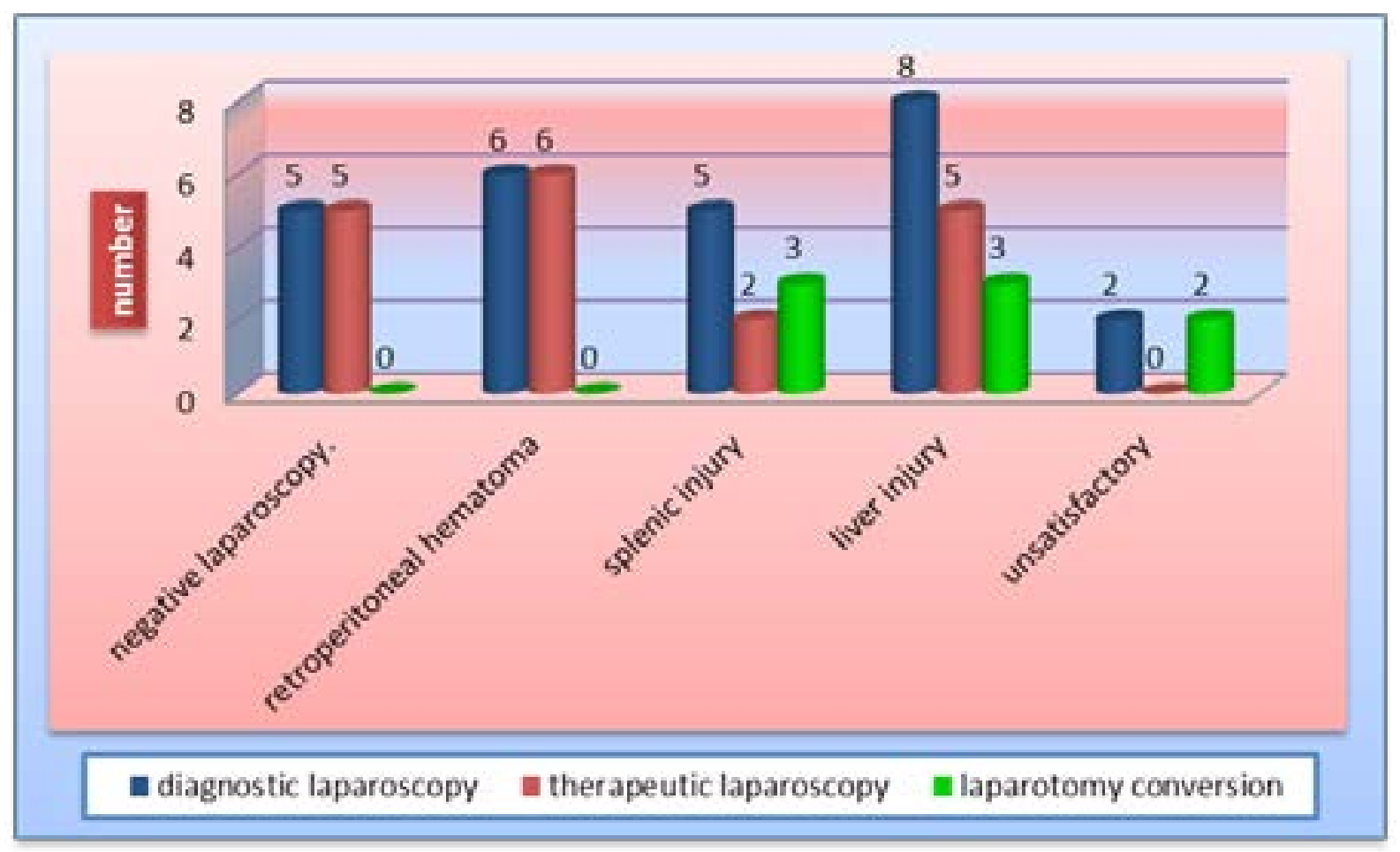

Fig 13: Types of injury and the procedure in blunt trauma patients.

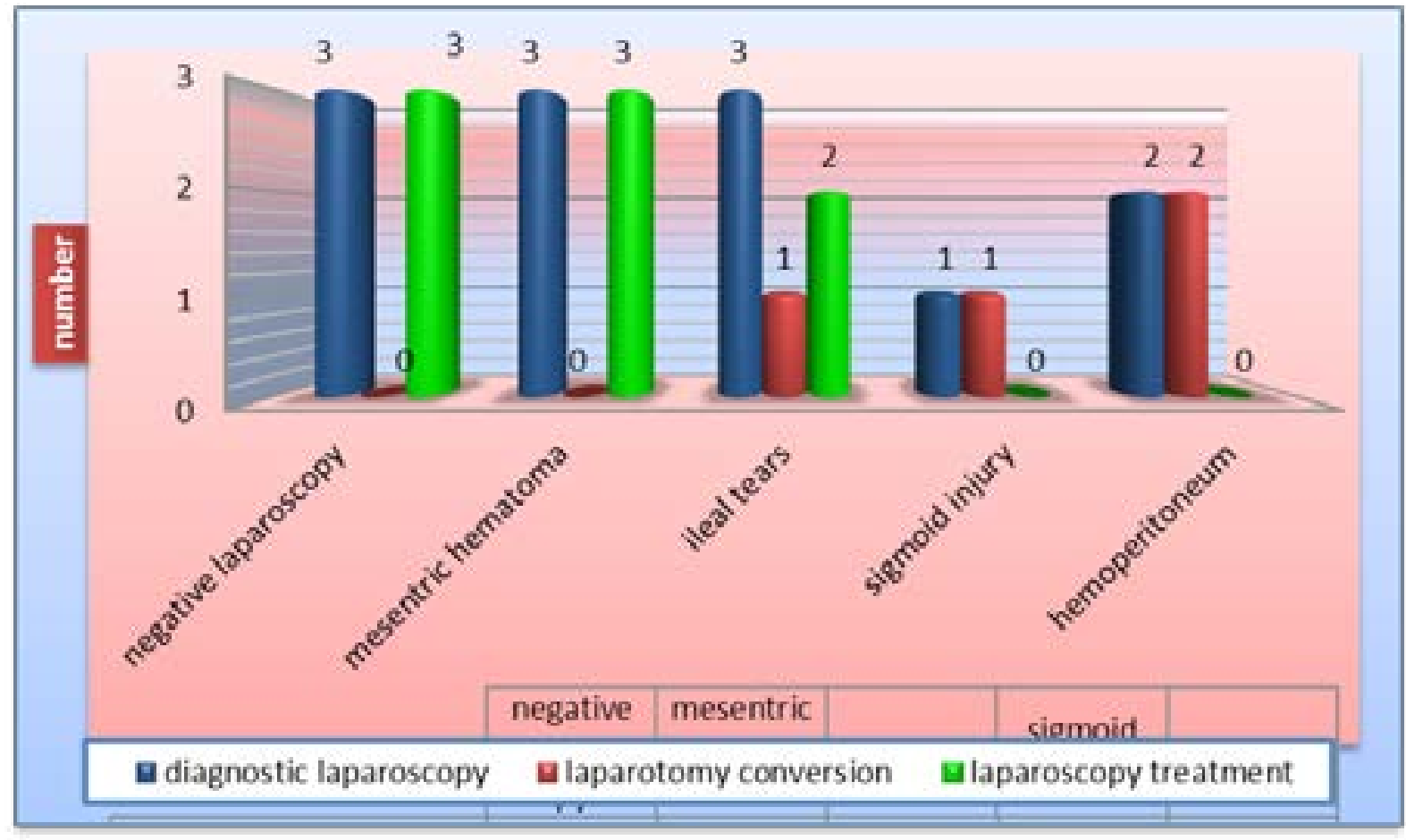

Fig 14: Types of injury and the procedure in sharp trauma patients. 


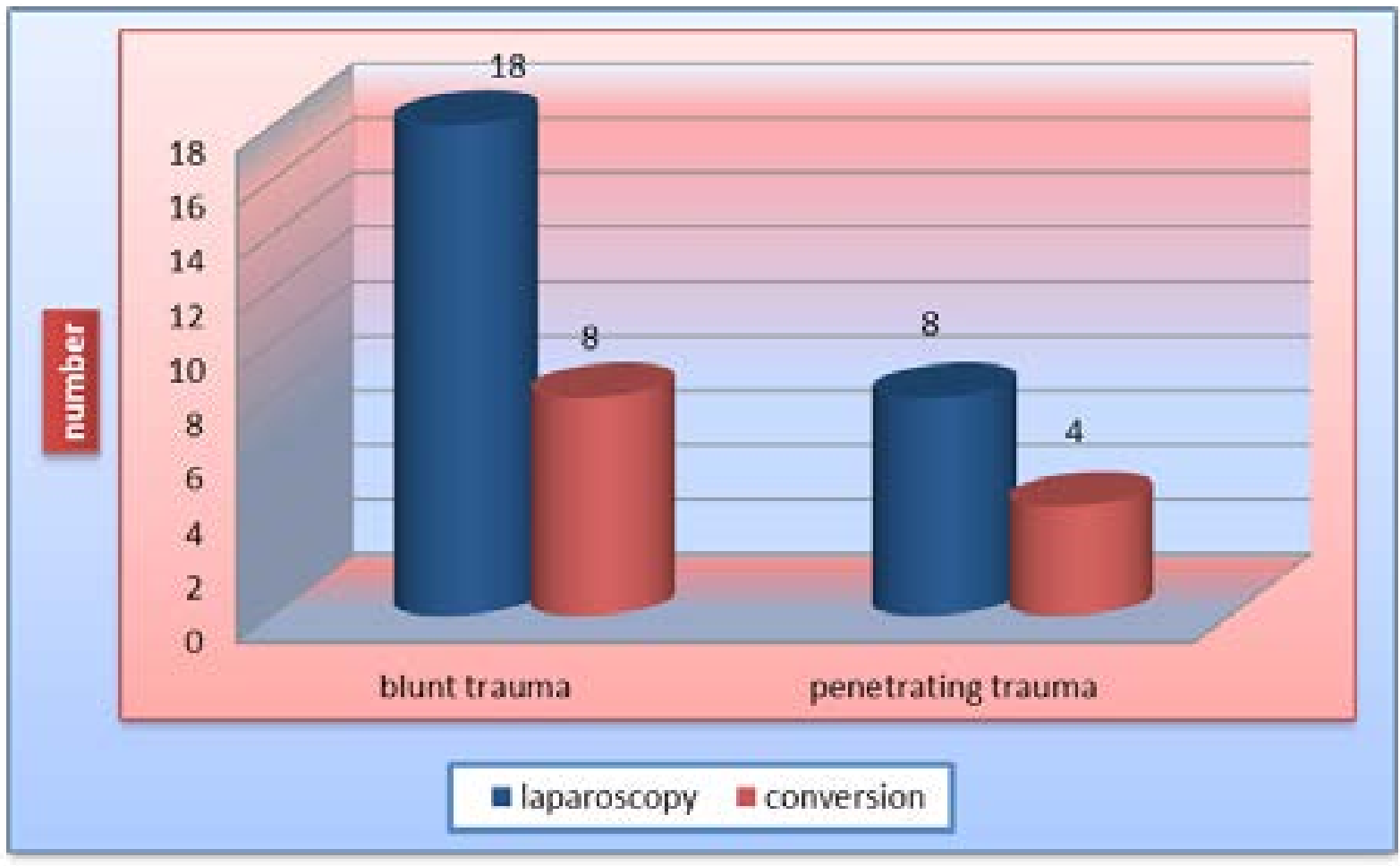

Fig 15: Laparoscopy versus conversion and type of trauma.

Table 3: Mean operative time

\begin{tabular}{lccc}
\hline & $\begin{array}{c}\text { Laparoscopy } \\
(\mathbf{n = 2 6 )}\end{array}$ & $\begin{array}{c}\text { Laparotomy conversion } \\
(\mathbf{n = 1 2})\end{array}$ & P value \\
\hline Operative time & $85 \mathrm{~min}$ & $67 \mathrm{~min}$ & $<0.005$ \\
\hline
\end{tabular}

Table 4: Postoperative pain assessment: Numeric Rating Scale (NRS)

\begin{tabular}{lccc}
\hline & $\begin{array}{c}\text { Laparoscopy } \\
(\mathbf{n = 2 6})\end{array}$ & $\begin{array}{c}\text { Laparotomy group } \\
(\mathbf{n = 1 2})\end{array}$ & P value \\
\hline NRS Range & $2-3$ & $5-7$ & $<0.001$ \\
\hline
\end{tabular}

Table 5: Hospital stay

\begin{tabular}{lccc}
\hline & $\begin{array}{c}\text { Laparoscopy } \\
(\mathbf{n = 2 6 )}\end{array}$ & $\begin{array}{c}\text { Laparotomy conversion } \\
(\mathbf{n = 1 2})\end{array}$ & P value \\
\hline Hospital stay & $2-5$ days & $6-8$ days & $<0.001$ \\
\hline
\end{tabular}

Table 6: Wound infection

\begin{tabular}{|c|c|c|c|}
\hline & $\begin{array}{l}\text { Laparoscopy } \\
(n=26)\end{array}$ & $\begin{array}{l}\text { Laparotomy conversion } \\
\qquad(\mathrm{n}=12)\end{array}$ & P value \\
\hline Wound infection & 0 & $3(25 \%)$ & $<0.003$ \\
\hline
\end{tabular}

Table 7: Incisional hernia

\begin{tabular}{lccc}
\hline & $\begin{array}{c}\text { Laparoscopy } \\
(\mathbf{n = 2 6 )}\end{array}$ & $\begin{array}{c}\text { Laparotomy conversion } \\
(\mathbf{n = 1 2})\end{array}$ & P value \\
\hline Incisional hernia & 0 & $1(8.3 \%)$ & $<0.02$ \\
\hline
\end{tabular}




\section{Discussion}

Recent trends in management of different forms of surgical problems are towards minimally invasive techniques. The current use of laparoscopy in diagnosis and management of trauma patients is a natural extension of this trend. Previously, there were literatures not supporting the use of laparoscopy in abdominal trauma due to the high incidence of complications, missed abdominal injuries, trocars' injuries and gas embolism. However, in the last decade this concept has been dramatically reduced due to the wide steps in development of laparoscopy and improvement of techniques and skills.

A study by Renz stated that exploratory laparotomy in either blunt or penetrating abdominal trauma with suspected intra-abdominal injuries is associated with a high negative (non-therapeutic) laparotomy rate and a high procedure-related morbidity (41\%). ${ }^{1}$ Another series showed that, diagnostic laparoscopy in trauma patient is reported to spare a median of $57 \%$ (range, 17$89 \%$ ) of non-therapeutic laparotomy. ${ }^{12}$

Some series concluded that abdominal exploration by laparotomy should not be considered as a worthy diagnostic and therapeutic procedure for patients with equivocal and unreliable findings, as it is associated with complication rates as high as $40 \%$, including $10 \%$ to $40 \%$ negative laparotomy rate, $20 \%$ morbidity rate, $0 \%$ to $5 \%$ mortality rate and $3 \%$ long-term risk of bowel obstruction. ${ }^{13}$

Our study included 26 patients with blunt trauma and 12 with sharp abdominal trauma, selected from the trauma patients presented to the emergency department, according to the selection criteria. Patients with blunt trauma are more suitable for laparoscopic management than those with sharp trauma, mostly related to severity of injuries and the hemodynamic instability. Also, most of the cases with blunt trauma were due to MCA $(65.3 \%)$, followed by falling from height $(27 \%)$ and lastly physical assaults (7.7\%). In sharp trauma, all of the cases were due to abdominal stabs. We subjected all patients to preoperative primary assessment with FAST, and pelvi-abdominal CT scans. The FAST was nonconclusive (unsatisfactory) in 7 cases with blunt trauma and in 2 cases with sharp trauma, while C.T. scan was non-conclusive in 3 cases with blunt trauma and 2 cases with sharp abdominal trauma. A study done by Schnüriger et al., implied that the FAST examination at the primary assessment failed to detect free fluid collections or organ lesions in 1 of every 5 patients with confirmed spleen or liver injury and it concluded that the low overall diagnostic sensitivity of FAST may lead to under estimated injury patterns and so delayed complications may occur. ${ }^{14}$

Berci et al reported that he had reduced the number of non-therapeutic laparotomies performed for hemo-peritoneum by $25 \%$ through the use of laparoscopy in 150 patients with blunt abdominal trauma. ${ }^{15}$

The rate of laparotomy conversion in our study is $29 \%$. The incidence of conversion is significantly different in both groups $(33.3 \%$ in the sharp penetrating trauma group compared to $27 \%$ in blunt trauma group). The causes of conversion in blunt trauma group were due to inaccessible liver tears that needed open repair and lacerated spleen that needed open splenectomy. The conversion in the penetrating trauma group was due to ileal loops perforations that needed resection and anastomosis and sigmoid colon injury that necessitated colonic diversion.

In some literatures, patients with high grade or actively bleeding splenic injuries were treated with laparoscopic application of collagen-fibrinogen human thrombin seal on oozing lacerations and if a major bleeding is encountered laparoscopic splenectomy is then performed. ${ }^{16,17}$

We did not use thrombin seal in cases with solid organs injuries due to the deficiency and the high cost. However, in minor liver or splenic tears, we could manage some cases by laparoscopic suturing and peritoneal irrigation and the others with just peritoneal irrigations to remove the accumulated blood. This was consistent with a study done by Letoublon, who showed that nonoperative management of hepatic and splenic injuries is successful in up to $80 \%$ of cases. ${ }^{18} \mathrm{Up}$ to $75 \%$ of these patients will demonstrate signs of inflammatory response due to the haemoperitoneum (fever, tachycardia and leukocytosis). The use of laparoscopy to remove the old blood from the peritoneal cavity could be accomplished safely and maybe beneficial, during these procedures the solid organs and the clots on their surface are left alone to avoid any potential haemorrhage.

In the present study the encountered cases with lacerated spleen were difficult to be managed laparoscopically and were time consuming and so we preferred laparotomy and formal splenectomy for patient's safety.

Some literatures demonstrated trauma cases presented with bile duct injuries and biliary peritonitis that could be treated with collagenfibrinogen human thrombin seal and/or drained laparoscopically. ${ }^{17,19}$ In the present study we did not encounter cases with biliary injuries.

Tears of the stomach, small bowel, and colon can 
be repaired laparoscopically, when an anastomosis or a long repair is required, these are usually performed extra-corporeally through a focused laparotomy (mini-laparotomy). ${ }^{20-22}$ These were consistent with our study, in which we performed laparotomy for multiple ileal lesions and colonic injury. However, Sitnikov et al., in their study concluded that, diagnostic and therapeutic video assisted laparoscopy can confidently be used in trauma centers as a diagnostic and therapeutic technique in patients with small bowel injuries. ${ }^{23}$ The later study also has shown significant accuracy, specificity, and sensitivity in triage and management of those patients. They stated that this technique allows early diagnosis of bowel injury, decreasing the time to definitive repair, and when combined with therapeutic laparoscopy reduces morbidity, mortality, hospital costs, and length of hospitalization associated with nontherapeutic open laparotomy.

The main concern about laparoscopy in trauma will always be directed towards missed injuries. A study concluded that, in order to minimize the risk of missing bowel injuries it might be worth to combine explorative laparoscopy with laparoscopic lavage based on Otomo's DPL criteria. This concept, which is definitely interesting although logistically demanding, warrants further evaluation. ${ }^{24,25}$

A large case series exist from institutions that provide full definitive laparoscopic management of any injuries (also in shocked and actively bleeding patients) with no or minimal missed injuries and low conversion rate. ${ }^{26,27}$

In our study, 8 cases (21\%) had negative laparoscopy (5 with blunt trauma and 3 with sharp trauma) and those patients were saved from nontherapeutic laparotomy. 19 cases (50\%) got benefit from therapeutic laparoscopy (14 cases with blunt trauma and 5 cases with sharp trauma). Johnson et $a_{1}{ }^{28}$ reported that, among 22 patients with blunt trauma who underwent diagnostic laparoscopy, laparoscopy was negative in 4 patients $(18.2 \%)$, non-therapeutic in 15 patients $(68.2 \%)$ and 3 patients $(13.6 \%)$ required conversion to an open procedure. In a similar study, negative explorations were reported in 19 trauma patients (47.5\%). ${ }^{29}$

When diagnostic laparoscopy has been used as a screening tool in abdominal trauma (conversion to laparotomy with the first encounter of a positive finding: peritoneal penetration in penetrating trauma or free blood in blunt trauma patients), the number of missed injuries was $<1 \% .^{30}$

In our study the sensitivity and diagnostic accuracy of laparoscopy in blunt trauma is $92.3 \%$ and $100 \%$ in penetrating trauma patients. The therapeutic value of laparoscopy reached $69 \%$ in blunt trauma patients while it reached $50 \%$ in penetrating trauma, these, to some extent, were consistent with a study for laparoscopy in penetrating trauma that reported a sensitivity of $80-100 \%$, specificity of $38-86 \%$, and accuracy of $54-89 \%,{ }^{31}$ also another study used laparoscopy in blunt trauma reported a sensitivity of $90-100 \%$, specificity of $86-100 \%$, and accuracy of $88-100 \%$ for bowel injuries. ${ }^{32}$ In another study, the sensitivity, specificity, and diagnostic accuracy of laparoscopy when used to predict the need for laparotomy range from 75 to $100 \% .^{30}$

The reported complications of laparoscopy in trauma included not only the usual complications of anesthesia and laparoscopy, but also some that are unique to the trauma patient. Fabian et $\mathrm{al}^{33}$ reported development of tension pneumothorax in patients with diaphragmatic injury, from positive pressure pneumo-peritoneum. They stopped the insufflations with immediate needle thoracocentesis followed by insertion of chest tube. The risk of gas embolism in patients with intra-abdominal venous injuries, especially liver lacerations, is a big problem. Smith et al, ${ }^{34}$ reported gas embolism encountered in two patients with injuries of the inferior vena cava tamponed by clot. This potential problem of laparoscopy has paved the interest in gasless laparoscopy through expansion of the peritoneal cavity by mechanical retractors. We did not include cases with pneumothorax in our study or encountered cases of gas embolism.

The postoperative pain shows statistic differences between the laparoscopy and laparotomy groups in the present study and revealed higher NRS in the latter group which reflects the more need of analgesics and narcotics.

The average length of the hospital stay in our study in the completed laparoscopic group was 3 days (range, 2 to 5 days) and was 5.2 days (range, 6 to 8 days) in the laparotomy converted group. This significant difference in the hospital stay was due to the difference in the NRS between both groups with its impact on the recovery period. These findings were consistent with the study done by Johnson et al. ${ }^{33}$ In which the mean length of hospital stay for patients with blunt injuries was 9 days for the laparoscopy completed group compared with 20 days for laparotomy converted group.

We encountered 3 cases (27.3\%) with wound infection in the laparotomy converted group, while no wound infection was detected in the totally laparoscopic group.

Also we have one case (9\%) of incisional hernia in 
the laparotomy converted group, while no similar detectable cases in the totally laparoscopic group.

We have no cases with missed injuries in our series as the laparoscopically unsatisfactory cases ( 2 cases with blunt trauma) were converted to laparotomy for patient's safety. No mortality cases were encountered due to proper selection of the patients. The use of Laparoscopy in trauma has cut down the hospital expenses in comparison with those patients who have had negative or nontherapeutic laparotomies.

Lee PC et al. ${ }^{35}$ In their study found out that diagnostic and therapeutic laparoscopy applied to carefully selected hemodynamically stable trauma patients proved to be safe and technically feasible.

For a long time mandatory surgical exploration for penetrating wounds to the abdomen has been a surgical dictum. Although non-operative management of blunt solid organ injuries and low-energy penetrating injuries is well established. It is acceptable only in a highly selected subset of hemodynamically stable adult patients without evidence of peritonitis. With the current laparoscopic technology, diagnostic laparoscopy is most efficacious for evaluation of equivocal penetrating wounds. However, patients with gunshot injuries to the abdomen warrant immediate laparotomy to control bleeding and the complex injuries.

\section{Conclusion}

The evaluation and management of abdominal trauma is multi-factorial and includes mechanism and location of injury, hemodynamic status of the patient, neurological status of the patient, associated injuries and institutional resources. Therefore, careful selection, high index of suspicion, and a low threshold for laparotomy will provide the patient the benefits of minimal invasive surgery and reducing the rates and morbidity of unnecessary laparotomy. As technology and surgeons expertise continues to improve, more standard therapeutic interventions may be done laparoscopically in the future.

\section{References}

1. Renz BM, Feliciano DV: Unnecessary laparotomies for trauma: A prospective study ofmorbidity. J Trauma 1995; 38:350-356.

2. Carnevale N, Baron N, Delaney HM: Peritoneoscopy as an aid in the diagnosis of abdominal trauma: a preliminary report. $\boldsymbol{J}$ Trauma 1977; 17: 634-641.

3. Gazzaniga AB, Slanton WEW, Bartlett RH: Laparoscopy in the diagnosis of blunt andpenetrating injuries to the abdomen. $\boldsymbol{A m} \boldsymbol{J}$ Surg 1976; 131: 315-318.

4. Cuschieri A, Hennessy TP, Stephens RB, Berci G: Diagnosis of significant abdominal trauma after road traffic accidents: preliminary results of a multicentre clinical trial comparing minilaparoscopy with peritoneal lavage. Ann. $\boldsymbol{R}$ Coll Surg Engl 1988; 70: 153-155.

5. Sido $B$, Grenacher $\mathrm{L}$, Friess $\mathrm{H}$, Bu" chler MW: Abdominal trauma. Orthopade 2005; 34: 880888.

6. Choi YB, Lim KS: Therapeutic laparoscopy for abdominal trauma. Surg Endosc 2003;17: 421427.

7. Cuesta M, Van Der Peet D, Veenhof A: Laparoscopic management of acute abdomen. Recent Advances in Surgery 31 ${ }^{\text {st }}$ ed 2008: 2743.

8. Uranus $\mathrm{S}$, Dorr K: Laparoscopy in Abdominal Trauma. Eur J. Trauma Emerg. Surg 2010; 36: 19-24.

9. Li Y, Xiang $Y$, Wu N, Wu L, Yu Z, et al: A Comparison of laparoscopy and laparotomy for the management of abdominal trauma: A systematic review and meta-analysis. World $\boldsymbol{J}$ Surg 2015; $39: 2862-2871$.

10. Uranus S, Popa DE, Diaconescu B, Schrittwieser R: Laparoscopy in penetrating abdominal trauma. World J Surg 2015;39 :1381-1388.

11. Marks JM, Youngelman DF, Berk T: Cost analysis of diagnostic laparoscopy versus laparotomy in the evaluation of penetrating abdominal trauma. Surg Endosc 1997; 11: 272-276.

12. Stefanidis D, Richardson WS, Chang L, Earle DB, Fanelli RD: The role of diagnostic laparoscopy for acute abdominal conditions: An evidence-based review. Surg Endosc 2009; 23: $16-23$.

13. Roos JE, Willmann JK, Platz A, Pfammatter T, Hilfiker PR, Marincek B: Multidetector CT: Detection of active hemorrhage in patients with blunt abdominal trauma. Am J Roentgenol 2002; 179: 437-444.

14. Schnüriger Beat, Joachim Kilz, Daniel Inderbitzin, et al: The accuracy of FAST in relation to grade of solid organ injuries: A retrospective analysis of 226 trauma patients with liver or splenic lesion. BMC Medical Imaging 2009; 9:3. 
15. Berci G, Sackier JM, Paz-Parlow M: Emergency laparoscopy. Am J Surg 1991; 161: 332-335.

16. Olmi S, Scaini A, Erba L, Bertolini A, Guaglio $M$, Croce $E$. Use of fibrin glue (Tissucol) as a hemostatic in laparoscopic conservative treatment of spleen trauma. Surg Endosc 2007; 21: 2051-2054.

17. Marzano E, Rosso E, Oussoultzoglou E, Collange $O$, Bachellier P, Pessaux P: Laparoscopic treatment of biliary peritonitis following nonoperative management of blunt liver trauma. World Journal of Emergency Surgery 2010; 5: 26.

18. Letoublon C, Chen $Y$, Arvieux C, Voirin D, Morra I, Broux C, Risse O: Delayed celiotomy or laparoscopy as part of the non-operative management of blunt hepatic trauma. World $\boldsymbol{J}$ Surg 2008; 32:1189-1193.

19. Sugrue M, Balogh Z, Lynch J, Bardsley J, Sisson $G$, Weigelt J: Guidelines for the management of haemodynamically stable patients with stab wounds to the anterior abdomen. $\boldsymbol{A N Z} \mathbf{J} \mathbf{S u r g}$ 2007; 77: 614-620.

20. Hope WW, Christmas AB, Jacobs Dg, Sing RF: Definitive laparoscopic repair of penetrating injuries to the colon and small intestine: $\boldsymbol{A}$ Case Report J Trauma 2009; 66: 931-932.

21. Iannelli $A$, Fabiani $P$, Karimdjee $B S$, Baque $P$, Venissac N, Gugenheim J: Therapeuticlaparoscopy for blunt abdominal trauma with bowel injuries. J Laparo Endosc Adv Surg Tech A 2003; 13:189-191.

22. Streck CJ, Lobe TE, Pietsch JB, Lovvorn HN III: Laparoscopic repair of traumatic bowel injury in children. $\boldsymbol{J}$ Ped Surg 2006; 41:1864-1869.

23. Sitnikov V, Yakubu A, Sarkisyan V, Turbin M: The role of video-assisted laparoscopy inmanagement of patients with small bowel injuries in abdominal trauma. Surg Endosc 2009; 23:125-129.

24. Otomo $Y$, Henmi $H$, Mashiko $K$, Kato $K$, Koike K, Koido Y, Kimura A, Honma M, Inoue J, Yamamoto $Y$ : New diagnostic peritoneal lavage criteria for diagnosis of intestinal injury. J Trauma 1998; 44: 131-139.
25. Vinces $F Y$, Madlinger RV: Laparoscopic exploration and lavage in penetrating abdominal stab wounds: A preliminary report. Ulus Trauma Acil Cerrahi Derg 2009; 15: 109 -12 .

26. Cherkasov M, Sitnikov V, Sarkisyan B, Degtirev O, Turbin M, YakubaA: Laparoscopy versus laparotomy in management of abdominal trauma. Surg Endosc 2008; 22: 228-231.

27. Lin HF, Wu JM, Tu CC, Chen HA, Shih HC: Value of diagnostic and therapeutic laparoscopy for abdominal stab wounds. World J Surg 2010; 34: 1653-1662.

28. Johnson J., Tabitha G., Alexander R., Joseph B. et al: The use of laparoscopy in the diagnosis and treatment of blunt and penetrating abdominal injuries: 10-year experience at a level 1 trauma center. Am J Surg 2013; 205: 317-720.

29. Mohammed S. Teamma: Evaluation of laparoscopy in the management of abdominal emergencies. Journal of the Arab Society for Medical Research 2013; 8: 19-25.

30. Hori Y: SAGES Guidelines Committee. Diagnostic laparoscopy guidelines. Surg Endosc 2008; 22: 1353-1383.

31. Villavicencio RT, Aucar JA: Analysis of laparoscopy in trauma. J Am Coll Surg 1999; 189: $11-20$.

32. Leppaniemi $A$, Haapiainen R: Diagnostic laparoscopy in abdominal stab wounds: A prospective, randomized study. J Trauma 2003; 54: 636-645.

33. Fabian TC, Croce MA, Stewart RM: A prospective analysis of diagnostic laparoscopy in trauma. Am Surg 1993; 217: 557-565.

34. Smith RS, Fry WR.,Morabito DJ., Koehler RH: Therapeutic laparoscopy in trauma. $\mathbf{A m} \mathbf{J}$ Surg 1995; 170: 632-637.

35. Lee PC, Lo C, Wu JM, Lin KL, Lin HF, Ko WJ: Laparoscopy decreases the laparotomy rate in hemodynamically stable patients with blunt abdominal trauma. Surg Innov 2014; 21: 155165. 